\title{
The Effect of Using Multiple Mathematical Representations of Rational number concepts in Basic stage Grades Students in Jordan
}

\author{
Khaled Ahmed Aqeel Alzubi
}

\section{ARTICLE INFO}

Article History:

Received 03.06.2020

Received in revised form

09.02.2021

Accepted

Available online 01.07.2021

\begin{abstract}
This study, aimed at the effect of using multiple mathematical representations of rational number concept in basic grades students in jordan. The current study employed the content analysis approach to investigate the multiple mathematical representations and transitions among them in 8th Grade Mathematics Textbook. An observation method was used to analyses the teacher practices ( $n=35$ observations), and record the representations and transitions. The results showed that there was an existence of symbol and verbal representations in the textbook and teachers' implementation. Meanwhile, the other three representations (pictures and figures, models and Cutters, and life situations). And This study explored the nature of difficulties of eighth-grade students who struggled to build their conceptual understanding of early fraction ideas. interviews with Pre and post of students were conducted for a sufficient identification of the nature of the students' difficulties. The study revealed The students also minimal use of informal ordering strategies that involve more conceptual than a procedural understanding of the concept of initial fraction ideas.
\end{abstract}

C IJERE. All rights reserved

Keywords:

Multiple mathematical representations, rational number concept, basic stage grades students in Jordan.

\section{INTRODUCTION}

Many international researchers who are interested in mathematics such as, Gagatsis and Shikalli (2004) mentioned that the more teachers use representations and their multiple transitions in their teaching, the more likely students able to understand and success in math. Moreover, Anthony and Walshaw (2009) while they reviewed many studies concerning representations and transitions found that the presentations link between different math ideas and concepts, which encourage students to understand the different connections among the concepts through discovering different representations of the concept. Hwang, Chen, Dung and Yang (2007) indicated that the improving students' skills in using multiple, and (Anshori. \& Suwidiyanti, 2020; Demirbağ,2020; Cai \& Lester, 2005; Dreher \& Kuntze, 2015; Dwi \& Subanji,2017; Eyong, Ugada \& Aminu, 2021; Vural \& Vural, 2021)

Therefore, this current study presents further evidence for at the effect of using multiple mathematical representations of rational number concept in basic grades students in jordan. The theoretical framework depends on the model which was constructed by (Lesh et al., 2003) for the math representations, as content analysis approach was used to explore the use of presentations and to investigate the various transitions in the textbook of 8th grade specifically the algebra unit which . Moreover, the significance of the current study also emerges from the use of qualitative method using observation tool to examine teacher's use of representations while teaching algebra unit and the extent to which they apply representations transitions according to (Lesh, 2003) model.

Reviewing the literature concerning mathematical representations; the work of Lesh, Post, and Behr (1987) was one of the most important in this area, they constructed a model of the various elements in which mathematical representations are structured. This model is consisted of five elements ; (1) representations by written symbols such as writing numbers or symbols to express the math concept, (2) spoken representations which indicate expressing the math concept by words which allows often for more rephrasing the words and linking the concepts with other areas and life situations. (3) Experience based interpreting representations by real-life context which use the daily life events that fit the math concept, (4) manipulative representations are using any tool that student can hold by hand such as models and cutters. Finally (5) representations by pictures or diagrams include pictures, drawings, and tables (Hidayanto \& Anwar, 2017; You \& Quinn, 2010) .Considering mathematical representations as the basic feature to teach and learn math, whereas researchers mentioned that mathematical representations are closely improving students' learning and understanding of math (Anthony \& Walshaw, 2009; Greeno \& Hall, 1997; Santulli, 2009; Tripathi, 2008). Nonetheless, there are

*khaledaa@hu.edu.jo, The Hashemite University, orcid.org/0000-0001-8647-4570 
only few studies that highlighted the role of the textbook and teachers in facilitating students' understanding through using mathematical representations (Bal, 2015; Bayazit, 2011; Yan \& Lianghuo, 2006; Ulu, 2017). Math representations are the key of their success in solving the math problems. Bayazit (2011) showed that most of the participants depend on the symbols representation of conjugation; and that the students' alternation between the iconic and algebraic representation of conjugation seems an important stage for developing the conjugation concept. Fraction concepts are one of the most challenging topics in basic and secondary school mathematics (National Mathematics Advisory Panel [NMAP], 2008; Siemon et al., 2015). Research studies indicate that many children have difficulties with the concept of early fraction ideas (Behr, Harel, Post \& Lesh, 1992; Carpenter, Coburn, Reys \& Wilson, 1976; Carpenter, Corbitt, Kepner, Lindquist, \& Reys, 1980; Hansen, Jordan, \& Rodrigues, 2017; Mack, 1995; Post, 1981; Siemon, Beswick, et al., 2015). These difficulties result in a lack of understanding about fraction concepts and fractional operations, which can lead to significant difficulties in algebra (Behr, Lesh, Post \& Silver, 1983). It is critical that the nature of these difficulties of early secondary students who struggle with the concept of early fraction ideas be explored, and that an earlier intervention be provided so that they have access to algebra and opportunities to be successful in mathematics. This study explored the nature of difficulties of seventh and eighth grade students who struggled to build their conceptual understanding of early fraction ideas, in particular, ordering fractions.

\section{Research Questions}

The study aimed to answer the following two questions:

1- What are the distributions of Lesh's network model of representations and their transitions in the unit of real numbers in the textbook of 8 th grade?

2- Is there an effect of using multiple mathematical representations of rational number concept in basic grades students in Jordan?

\section{METHODS}

The current study employed the content analysis approach to investigate the multiple mathematical representations and transitions among them in the textbook of 8th grade due to the importance of the eighth grade as the Ministry of Education in Jordan deems a stage through which it can enable students assimilate the different math concepts. Furthermore, the TIMSS exams are made on the grade, because there is a similarity in the content of the Jordanian, European, and American mathematics textbooks for the eighth grade in the fields of numbers, geometry, algebra, statistics and probabilities, as they are all built in light of the NCTM. The researcher argues that content analysis will provide a clear image of multiple mathematical representations and transitions among them as it illustrated the data in details. In addition, the observation tool was also used to examine the extent of the math teachers' use of these representations and transitions in their classes. The researcher visited the jaresh educational district. He asked for one teacher from each school for his observation that has to have BA degree in Maths and teach 8th grade classes for at least 5 years. After having the names of teachers, contacted them personally to ask them for their agreement on participation. Three male teachers and two female teachers were the chosen participants who were coded by their first letter of their names and took numbers from 1-5. This study is a teaching experiment design research. The researchers performed the role of the teachers in the facilitation of student and problem interactions. This methodology is appropriate because the intention of the study is to explore the influence of concrete and pictorial models on student understanding (Lesh \& Kelly, 2000). The setting of this research study was a 8th grade in jaresh with close to (75) students, 25 male 50 female. The schools did not make Adequate Year Progress TIMSS in Mathematics.

The participants in this study were (Group A: four females) Group B: two males and two females) mathematics level or below and have shown particular deficiencies in their understanding of fractions in their class work and district-wide assessments, the MAP Tests. In reporting from this study pseudonyms were used to protect students' identity.

The intervention consisted of a sequence of lessons adapted from the Rational Number Project: Initial Fraction Ideas (Cramer et al., 2009a) curriculum. The lessons involved the use of fraction circles and 
focused on ordering fractions. Each group of students engaged in a weekly class session for 35 minutes of class time. The researcher worked with each group of students as they engaged in the lessons and activities for four weekly class sessions. Qualitative methodology was pursued for this study. Pre and post group interviews were conducted with eachstudent group for a dual purpose: for a sufficient identification of the nature of the students' difficulties and for an exploration of influence of the intervention. The primary sources of data for this study were transcripts of student group interviews that were video and audiorecorded, student work samples and researcher field notes. The interviews followed interview outlines from the Rational Number Project (RNP) curricula (Cramer et al., 2009a; Cramer et al., 2009b). The RNP curriculum lessons, student activities and interview protocols are designed to provide students opportunities to work flexibly within the different representations. As mentioned before, the framework outlined in Hiebert and Carpenter's (1992) theory of understanding and the Lesh Translation Model (Lesh et al., 1987) serve as the conceptual framework used to analyze the qualitative data gathered in this study. The data analysis focused on the representations in the manipulative aids (fraction circle), pictures (fraction circles or strips), symbols, language, and real-world situations, as well as transfer among and within these representations. The data analysis was conducted by combining two coding strategies - starting with some preset categories (codes) and adding others as they become apparent from the data (Corbin \&Strauss, 2008; Miles \& Huberman, 1994). The list in Table 3 was used to code the data for this study.

The data were organized into the categories, and the number of times that each student strategy came up was counted to figure out general patterns in the data. The frequency table reveals a rough estimate of relative importance and subtle differences within and between the categories or subcategories (see Table 4). The type of table including frequency counts is not suited to statistical analysis, but it reveals general patterns in the data (Miles \& Huberman, 1994). The researcher developed a table that contained all the determined variables of the five elements of Lesh's network model and their transitions, and then the research asked other two colleges to develop a table to the same topic. The consistency of the data obtained created the basic for ensuring validity and reliability. A total of 7 lessons and 7 class visits were accessed by first semester of 2018/2019 and which have been analyzed researcher who used the prepared table. Namely, representation in written symbols $(\mathrm{R})$, verbal representation $(\mathrm{L})$, real life situation representations $(\mathrm{H})$, manipulative representation $(\mathrm{N})$, and pictures and diagrams representation (S). The tables were compared to identify the differences among the related representations and their transitions and which were examined again. Moreover, the comparison allowed obtaining common themes, which strengthened the validity and reliability of the table that was used in this study. Finally, for ensuring the interpretative validity, the researcher let other math supervisor's members who supervise teachers to read the initial findings to give any feedback of incorrect representations.

\section{Data Analysis}

1 -Analysis the real numbers unit in eighth grade math textbook, calculation of the percentages of the representations, as well as the transition amount among the applied representations.

2 -A ttend all lessons on the topic ofreal numbers for every participant, calculation of the percentage of each of the math representations and transitions among them. Thereafter, comparing them with textbook representations and transitions, on one hand, and with Lesh et al. (2003) model on the other, as an attempt to link between what is theoretically stated in the textbook and what is going inside the classroom.

The current study used a specific method of qualitative research - content analysis- which categorizes the raw data by developing a descriptive data such as percentages and frequencies to reach a conclusion, and this could limit the comparison with other qualitative research methods. Moreover, this study examined the real numbers unit in the 8th standardized textbook grade in Jordan the thing which may influence the applicable features in other contexts, therefore, generalization of the findings will not be encouraged to make.

\section{RESULTS}

The results of the transitions of the representations showed that transition from the written symbol to the verbal representation has the highest percentage $(29 \%)$, followed by transition from verbal to written symbol representation with percentage $(24 \%)$ with a low level of distribution. Percentage of the transition 
from pictures and diagrams to written symbol representation is in the lower level(19\%), as the percentage of the transition from verbal to pictures and diagrams representation $(9 \%)$ and the percentages ofthe other transitions which were ranged between ( $1 \%-4 \%)$ very low. Moreover, results of low representation by real life situations are in agreement with the results of the study of Yan and Lianghuo (2004) which cited that it is a common in most textbooks in the world the lack of real life situations representations such as the Unites States of America and China. Results of this study also align with the study of Bal (2015) that there is a lack in the use of representations in the curricula; and that the presentation of the curricula is still controlled by the traditional way. Showed that students' difficulties with the concept of early fraction ideas persist. These persistent difficulties of early fraction ideas were due to a lack of the students' understanding of the partwhole and ratio subconstructs of fractions. The students mainly relied on whole number thinking for ordering fractions. The students revealed minimal use of informal ordering strategies that reflect their use of mental images of fractions to judge the fraction's relative size. Even after the four weeks of instruction, the students' overgeneralization of whole number thinking to the field of rational numbers was still the main factor related to their difficulties with the concept of ordering fractions. The whole number dominance strategies were often used by the students for ordering fractions before and even after the remediation intervention.

However, there was subtle (but meaningful) evidence that the interventionusing multiple mathematical representations using the fraction circle model had a positive influence on the students' understanding of fraction concepts: an increasing use of informal ordering strategies and a decreased reliance on whole number thinking. A limitation of this study is the short intervention time period. The instruction for the four weekly class sessions was not enough for the students to overcome their whole number thinking. There were not any clear effects of the intervention (at least in the statistical sense) on the students' understanding of fraction concepts.

Q1-What are the distributions of Lesh's network model of representations and their transitions in the unit of real numbers in the textbook of 8 th grade?

Using the rich analysis of Lesh et al. (2003) network model in the level of distribution of math representations and their transitions, which is categorized into four levels, level 1 is considered lower level of presentation (less than 0.20 ), level 2 is a low level (between (0.20- less than 0.40); level 3 is a medium level between (0.40-0.60); level 4 is a high level between $(0.60$ - less than 0.80$)$ and very high is the level 5 (more than 0.8 ). The results of the current study revealed that there are about 374 representations in the unit, written symbols and verbal representations were in the medium level (38.24-38.50), whereas, real life situations and manipulative and pictures and diagrams were in the lower level (2.94-10.70). Meanwhile, the results of the transitions of the representations showed that transition from the written symbol to the verbal representation has the highest percentage $(29 \%)$, followed by transition from verbal to written symbol representation with percentage $(24 \%)$ with a low level of distribution. Percentage of the transition from pictures and diagrams to written symbol representation is in the lower level $(19 \%)$, as the percentage of the transition from verbal to pictures and diagrams representation $(9 \%)$ and the percentages ofthe other transitions which were ranged between $(1 \%-4 \%)$ very low.

The above mentioned revealed that the average of the transitions among the different representations was in the lower level, Figure 3 illustrates the percentages of the math textbook distribution of the multiple representations and the transitions among them. For instance, transition percentage from pictures and diagrams representation to manipulative representation was $(4 \%)$, while transitions percentage from manipulative representation to pictures and diagrams representation were (2\%) Despite, the results showed that the distributions of written symbols and verbal representations were in the medium level; the percentages of the of transitions were low, This reveals the fact that the multiple math representations were taught separately and not in a specific math content which affect students' understanding and learning and this align with the finding of Greeno and Hall (1997) who stated that teaching the multiple types of representations separately and not in a connected ways would not structure students' knowledge and engage them in meaningful and continuous ways. Juersivich, Garofalo and Fraser (2009) mentioned that the use of the representations as ends by themselves is not a functional in improving students' understanding. 
Moreover, results of low representation by real life situations are in agreement with the results of the study of Yan and Lianghuo (2004) which cited that it is a common in most textbooks in the world the lack of real life situations representations such as the Unites States of America and China. Results of this study also align with the study of Bal (2015) that there is a lack in the use of representations in the curricula; and that the presentation of the curricula is still controlled by the traditional way. The researcher revised the outlines of the Jordanian math curriculum and its objectives, where he found that the call of using the multiple representations such as tables, shapes, pictures and writing symbols. Other objectives were found which highlight the importance of math in the daily life, and acquiring math knowledge that helps the individual in his/her life. The results of the analysis showed a shortcoming in the math representations, as stated earlier, which the Ministry of Education in Jordan works to provide in the math curricula.

The researcher noted also that the concepts and activities are widely and repeatedly presented in the same representations; that there is an increase in the number of activities; and that this amount of activities burdens both the learners and teachers. This result is in agreement with those provided in the study of Yan and Lianghuo (2006) in the junior secondary stage schoolbooks in the United States of America. The researchers noted that the math books in the USA contain math problems much more than those in China, which burdens the learner. In this concern, the researcher suggests decreasing the number of activities related to the same idea, and focus on presenting the idea or concept in more than one of these representations, especially real life situations. It is also suggested to use suitable transitions among these representations and encourage the students to justify their understandings. In the light of these results, the researcher finds that one of the problems in math teaching-learning process is the shortage in the use of math representations, particularly real life situations, This view is in line with the study of Leinwand and Ginsburg (2007), who noted that the math textbooks in Singapore use multiple g math representations and transitions among them, to a wider scale, more than their use in the United States of America. In their point of view, this is the reason for the success of math curricula in Singapore.

Q2- Is there an effect of using multiple mathematical representations of rational number concept in basic grades students in Jordan?

This study identified the whole number dominance strategies used by the students for ordering fractions before and even after the intervention. The analysis of the interview data shows that the students in both Group A and Group B frequently relied on whole number thinking for ordering fractions . The overgeneralization of whole number thinking to the field of rational numbers is one of reasons for why the students' difficulty with the concept of fraction order persists. Another finding from the analysis of the interview data is that the students revealed minimal use of informal ordering strategies that reflect their use of mental images of fractions to judge the fraction's relative size. The informal strategies for ordering fractions have been identified by the Rational Number. When using the four strategies: same numerator, same denominator, transitive and residual strategies, students use their mental images of fractions to judge the relative size of the fractions. Thus, the informal strategies involve more conceptual than procedural understanding of the concept of initial fraction ideas (Cramer et al., 2009a). However, our interview data showed that the students infrequently used the informal strategies for ordering fractions. They seldom constructed the informal ordering strategies nor did they usemental images of fractions. An additional interesting fact shown in the Table 4 is that the students showed little reliance on the procedural strategies usually taught for ordering fractions as well as the informal strategies.

Even though the whole number thinking still dominated after the intervention. The frequencies of whole number thinking (34\%) and informal strategies (33\%) used by the students in Group A are almost the same. In addition, the decrease of the fraction strips use (from $30 \%$ to $4 \%$ ) became apparent in the data from Group A. Before the intervention, there was an evidence for misleading of the fraction strips use to whole number thinking. For example, the students considered the denominators as the number of "rooms" in their fraction strip model (they called the pieces divided from a whole "rooms") and finally focused on the total number of "rooms" colored according to the numerators for comparing two fractions. They considered the numerators and denominators as four independent numbers then treated them like whole numbers. Note that the term "room" is linked to whole number. The decrease of the fraction strips seems to linked to the decrease of whole number thinking (from $45 \%$ to $34 \%$ ) and the relative increase of informal strategies use by the students in Group A (from 6\% to 33\%). This finding indicates that students should be carefully taught 
with even concrete models, such as fraction strips. The lack of understanding of how to use concrete models could interfere with the development of understanding of fraction concepts. Finally, the excerpt below shows how a student in Group A used an informal strategy, the "transitive" strategy, to identify an order relation of fractions.

\section{Conclusions and Discussion}

The analysis of interviews with the students who struggle showed that their difficulties with the concept of early fraction ideas persist. These persistent difficulties of early fraction ideas were due to a lack of the students' understanding of the part-whole and ratio subconstructs of fractions. The students mainly relied on whole number thinking for ordering fractions. The students revealed minimal use of informal ordering strategies that reflect their use of mental images of fractions to judge the fraction's relative size. Even after the four weeks of instruction, the students' overgeneralization of whole number thinking to the field of rational numbers was still the main factor related to their difficulties with the concept of ordering fractions. The whole number dominance strategies were often used by the students for ordering fractions before and even after the remediation intervention. However, there was subtle (but meaningful) evidence that the intervention using the fraction circle model had a positive influence on the students' understanding of fraction concepts: an increasing use of informal ordering strategies and a decreased reliance on whole number thinking. A limitation of this study is the short intervention time period. The instruction for the four weekly class sessions was not enough for the students to overcome their whole number thinking. There were not any clear effects of the intervention (at least in the statistical sense) on the students' understanding of fraction concepts. This might be due to the short intervention time period. The students need more time with concrete models, such as fraction circles, to develop mental images and a conceptual understanding of fractions. This limitation provides opportunities for future research by suggesting studies to investigate the influence of a remediation intervention with enough time period (e.g., at least more than the total of 7 hours that is about three times the intervention time period of the current study) using the fraction circle model on students' understanding of fraction concepts. Further research, such as a larger-scale quantitative research, is required for generalizability.

Concrete models help students develop their own understanding of fraction concepts based on their existing knowledge, such as their knowledge of whole numbers. However, the misleading use of the fraction strips that supported their whole number thinking illustrated how an incomplete understanding of a concrete model could interfere with the concept of fraction order. Lessons and activities that involve the use of concrete models with a complete understanding of them would show promise for effective remediation processes that focus on the development of mental images regarding fractions, and ultimately, a conceptual understanding of fraction concepts. Further research is also needed to investigate how to effectively support students' complete understanding of the use of concrete models using appropriate and accurate language.

This study suggests the need of a remedial intervention for students showing the persistent difficulty with early fraction ideas. Students need to be given enough time with not only concrete models but also appropriate usage of language to support a complete understanding of how to use the models. An earlier intervention involving the use of different models for connections among multiple representations should also be considered for students to develop their conceptual understanding of fraction concepts. The administration at the Ministry of Education in Jordan may benefit from the current study results concerning issues related to the curricula improvement and development. Also the Researchers could use the tools of this study for future research. Comparisons could be conducted with other studies using content analysis approach. The current study may attract the attention of math teachers and supervisors to the importance of using the multiple representation and their transitions.

\section{REFERENCES}

Anshori,I. \& Suwidiyanti (2020). Improvement of mathematics teacher performance through academic supervision with collaborative approaches. International Journal of Educational Research Review,4(3),227-242.

Anthony, G., \& Walshaw, M. (2009). Characteristics of effective teaching of mathematics: A view from the West. Journal of Mathematics Education, 2(2), 147-164. 
Bal, A. (2015). Skills of using and transform multiple representations of the prospective teachers. Journal of Mathematical Behavior, 197(2015), 582-588. https://doi.org/10.1016/j.sbspro.2015.07.197.

Bayazit, I. (2011). Prospective teachers' inclination to single representation and their development of the function concept. Educational Research and Reviews, 6(5), 436- 446.

Cai, J., \& Lester, F. (2005). Solution Representations and pedagogical representations in Chinese and U.S. classrooms. Journal of Mathematical Behavior, 24(3), 221-237. https://doi.org/10.1016/j.jmathb.2005. 09.003.

Demirbağ,T .(2020). Views of students and teachers about role of homework implementation on students' academic success. The Universal Academic Research Journal,2(2),64-77.

Doerr, H., \& Lesh, R. (2011). Models and modelling perspectives on teaching and learning mathematics in the Twenty-First Century. In Trends in teaching and learning of mathematical modelling (pp. 247-268). Springer, Dordrecht. https://doi.org/10.1007/978-94-007-0910-2_26.

Dreher, A., \& Kuntze, S. (2015). Teachers' professional knowledge and noticing: The case of multiple representations in the mathematics classroom. Educational Studies in Mathematics, 88(1), 89114.https://doi.org/10.1007/s10649-014-9577-8.

Dwi. R, Subanji, P, Hidayanto, E., \& Anwar, R. (2017). Process of mathematical representation translation from verbal into graphic. Mathematic Education, 12(4), 367-381.

Eyong,E,I., Ugada,C. \& Aminu,A. (2020). Indicators of improved achievement of students' in mathematics. The Universal Academic Research Journal,2(1), 29-37.

Gagatsis, A., \& Shiakalli, M. (2004). Ability to translate from one representation of the concept of function to another and mathematical problem solving. Educational Psychology, 24(5), 645-657. https://doi.org/10.1080/0144341042000262953.

Greeno, J., \& Hall, B. (1997). Practicing representation: Learning with and about representational forms. Phi Delta Kappa International, 78(5), 99-107.

Hariyani,S. Ahmad,N.J.\&Marsitin,R. (2021). Mathematics teaching practicum for junior high school in a different culture-based situation. The Universal Academic Research Journal,3(2),77-86.

Hwang, W., Chen, N., Dung, J., \& Yang, Y. (2007). Multiple representation skills and creativity effects on mathematical problem solving using a multimedia whiteboard system. Educational Technology E Society, 10(2), 191-212.

Juersivich, N., Garofalo, G., \& Fraser, V. (2009). Student teachers' use of technology- generated representations: Examples and rationales. Journal of Technology and Teacher Education, 17(2), 149-173.

Koretsky, M., Keeler, J., Ivanovitch, J., \& Cao, Y.(2018), The role of pedagogical tools in active learning: a case for sense-making. International Journal of STEM Education, 5(18), 2-20. https://doi.org/10.1186/s40594-018-01165.

Leinwand, S., \& Ginsburg, A. (2007). Learning from Singapore Math: The United States could benefit from looking at five elements driving the program's success. Educational Leadership, 65(3), 32-36.

Lesh, R, Post, T. \& Behr, M. (1987). Representations and translations among representations in mathematics learning and problem solving. Retrieved on 28/2/2018 from http://www.cehd.umn.edu/ rationalnumberproject/87_5.html. 
Lesh, R., \& Harel, G. (2003). Problem solving, modeling, and local conceptual development. Mathematical thinking and learning, 5(2-3), 157-189. https://doi.org/10.1080/10986065.2003.9679998.

Lesh, R., \& Lehrer, R. (2003). Models and modeling perspectives on the development of students and teachers. Mathematical Thinking and Learning, 5(2\&3), 109-130. https://doi.org/10.1080/10986065.2003.9679996.

Lesh, R., Cramer, K., Doerr, H., Post, T. \& Zawojewski, J. (2003). Using a translation model for curriculum development and classroom instruction. Retrieved on 5/1/2018, from: http://www.cehd.umn.edu/rationalnumberproject/03_1.html.

Lesh, R., Post, T., \& Behr, M. (1987). Representations and translations among representations in mathematics learning and problem solving. In C. Janvier, (Ed.), Problems of representations in the teaching and learning of mathematics (pp. 33 - 40). Hillsdale, NJ: Lawrence Erlbaum.

Mack, N. (1990). Learning fractions with understanding: Building on informal knowledge. Journal for Research in Mathematics Education, 21, 16 - 32.

Mack, N. K. (1995). Confounding whole-number and fraction concepts when building on informal knowledge. Journal for Research in Mathematics Education, 26, 422-441.

Miles, M. B., \& Huberman, A. M. (1994). Qualitative data analysis. Thousand Oaks, CA: Sage.

Moss, J. \& Case, R. (1999). Developing children's understanding of the rational numbers: A new model and an experimental curriculum. Journal for Research in Mathematics Education, 30, 122 - 147.

National Mathematics Advisory Panel (2008). Foundations for success: The final report of the national mathematics advisory panel. Washington D.C.: U. S. Department of Education.

Post, T., Behr, M., \& Lesh, R. (1982). Interpretations of rational number concepts. In L. Silvey \& J. Smart (Mathematics for Grades 5-9, 1982 NCTM Yearbook (pp. 59-72). Reston, Virginia: NCTM.

Post, T., Wachsmuth, I., Lesh, R., \& Behr, M. (1985). Order and equivalence of rational numbers: A cognitive analysis. Journal for Research in Mathematics Education, 16, 18-36.

Santulli, T. (2009). Representations from the Real World. Mathematics teaching in the middle school, 14(8), 466473.

Siemon, D., Beswick, K., Brady, K., Clark, J., Faragher, R., \& Warren, E. (2015). Teaching mathematics: foundations to middle years (2nd Edition). South Melbourne, Victoria: Oxford University Press.

Silver, E. (1986). Using conceptual and procedural knowledge: A focus on relationships. In J. Hiebert (Conceptual and procedural knowledge: The case of mathematics (pp. 181 - 198). Hillsdale, NJ: Lawrence Erlbaum Associates.

Treffert-Thomas, S., Viirman, O., Hernandez-Martinez, P., \& Rogovchenko, Y. (2017). Mathematics lecturers' views on the teaching of mathematical modelling. Education, 22(4), 121-145.

Tripathi, P. (2008). Developing mathematical understanding through multiple representations. Mathematics Teaching in Middle School, 13(89), 438-445.

U.S. Department of Education (2010). National assessment of educational progress. National Center for Educational Statistics. Retrieved from http://nces.ed.gov/nationsreportcard/. 
Ulu, M. (2017). Examining the mathematical modeling processes of primary school 4th-grade students: Shopping problem. Universal Journal of Educational Research, 5(4), 561-580. https://doi.org/10.13189/ujer.2017.050406.

Vural,Ö.F. \& Vural,S. (2021). An examination of 5th grade mathematics curriculum in terms of 21st century skills. International Journal of Educational Research Review,6(2),82-92.

Yan, Z., \& Lianghuo, F. (2006). Focus on the representation of problem types in intended curriculum: A comparison of selected mathematics textboks from maibland China and The United States. International Journal of Science and Mathematics Education, 4(4), 609-626. https://doi.org/10.1007/s10763-006-9036-9.

You, Z., \& Quinn, R. (2010). Prospective elementary and middle school teachers' knowledge of linear functions: A quantitative approach. Journal of Mathematics Education, 3(1), 66-76. 